\title{
Clinical implications of incidental venous thromboembolism in cancer patients
}

\author{
Frits I. Mulder ${ }^{1,2}$, Marcello Di Nisio ${ }^{3}$, Cihan Ay ${ }^{4}$, Marc Carrier ${ }^{5}$, Floris T.M. Bosch ${ }^{1,2}$, \\ Annelise Segers ${ }^{6}$, Noémie Kraaijpoel ${ }^{1}$, Michael A. Grosso ${ }^{7}$, George Zhang ${ }^{7}$, \\ Peter Verhamme ${ }^{8}$, Tzu-Fei Wang ${ }^{9}$, Jeffrey I. Weitz ${ }^{10}$, Saskia Middeldorp (1)', \\ Gary Raskob ${ }^{11}$, Ludo F.M. Beenen (10 ${ }^{12}$, Harry R. Büller ${ }^{1}$ and Nick van Es ${ }^{1}$
}

\begin{abstract}
Affiliations: ${ }^{1}$ Dept of Vascular Medicine, Amsterdam Cardiovascular Science, Amsterdam UMC, University of Amsterdam, Amsterdam, The Netherlands. ${ }^{2}$ Dept of Internal Medicine, Tergooi Hospitals, Hilversum, The Netherlands. ${ }^{3}$ Dept of Medicine and Ageing Sciences, University G. D'Annunzio, Chieti, Italy. ${ }^{4}$ Dept of Medicine I, Clinical Division of Haematology and Haemostaseology, Medical University of Vienna, Vienna, Austria. ${ }^{5}$ Dept of Medicine, Ottawa Hospital Research Institute at the University of Ottawa, Ottawa, ON, Canada. ${ }^{6}$ ITREAS, Academic Research Organization, Amsterdam, The Netherlands. ${ }^{7}$ Daiichi Sankyo Pharma Development, Basking Ridge, NJ, USA. ${ }^{8}$ Dept of Vascular Medicine and Hemostasis, University Hospitals Leuven, Leuven, Belgium. ${ }^{9}$ Dept of Internal Medicine, Division of Hematology, The Ohio State University Wexner Medical Center, Columbus, OH, USA. ${ }^{10}$ McMaster University and the Thrombosis and Atherosclerosis Research Institute, Hamilton, ON, Canada. ${ }^{11}$ University of Oklahoma Health Sciences Center, College of Public Health, Oklahoma City, OK, USA. ${ }^{12}$ Dept of Radiology, Amsterdam UMC, University of Amsterdam, Amsterdam, The Netherlands.
\end{abstract}

Correspondence: Frits I. Mulder, Dept of Vascular Medicine, Amsterdam Cardiovascular Science, Amsterdam UMC, University of Amsterdam, Meibergdreef 9, 1105 AZ Amsterdam, The Netherlands.

E-mail: f.i.mulderdamc.nl

@ERSpublications

In cancer patients with incidental venous thromboembolism (VTE), the risk of recurrent VTE is substantial despite initiation of anticoagulant treatment. Incidental VTE is a serious condition, which should be treated similar to symptomatic VTE. http://bit.ly/2qM4XUp

Cite this article as: Mulder FI, Di Nisio M, Ay C, et al. Clinical implications of incidental venous thromboembolism in cancer patients. Eur Respir J 2020; 55: 1901697 [https://doi.org/10.1183/ 13993003.01697-2019].

\section{ABSTRACT}

Introduction: In cancer patients, current guidance suggests similar treatment for incidental and symptomatic venous thromboembolism (VTE), mainly based on retrospective data. We aimed to evaluate anticoagulant therapy in cancer patients with incidental and symptomatic VTE.

Methods: The Hokusai VTE Cancer Study was a randomised controlled trial comparing edoxaban with dalteparin for cancer-associated VTE. The primary outcome was the composite of first recurrent VTE or major bleeding. Secondary outcomes included major bleeding, recurrent VTE and mortality. Outcomes in patients with incidental and symptomatic VTE were evaluated during the 12-month study period.

Results: 331 patients with incidental VTE and 679 patients with symptomatic VTE were enrolled, of whom the index event was confirmed by an independent radiologist. Median durations of anticoagulant treatment were 195 and 189 days, respectively. In patients with incidental VTE, the primary outcome occurred in $12.7 \%$ of patients, major bleeding in $6.6 \%$ of patients and recurrent VTE in $7.9 \%$ of patients. Out of the 26 VTE recurrences in patients with incidental VTE, five (31\%) were incidental, seven (44\%) were symptomatic and four (25\%) were deaths for which pulmonary embolism could not be ruled out. In patients with symptomatic VTE, the primary outcome occurred in $13.8 \%$ of patients, major bleeding in $4.9 \%$ of patients and recurrent VTE in $10.9 \%$ of patients. All-cause mortality was similar in both groups.

Conclusion: Clinical adverse outcomes are substantial in both cancer patients with incidental and symptomatic VTE, supporting current guideline recommendations that suggest treating incidental VTE in the same manner as symptomatic VTE. 


\section{Introduction}

In clinical practice $\sim 20 \%$ of all VTE events are related to cancer [1]. VTE can manifest as deep-vein thrombosis (DVT) and/or pulmonary embolism, and impact a cancer patient's disease journey by increasing morbidity and the risk of mortality [2,3]. With improvements in computed tomography scanning technology, and its increased utilisation for diagnosis and monitoring of cancer, the proportion of cancer patients with incidental VTE has risen over time [4-7]. Nowadays, about half of all pulmonary embolic events in cancer patients are classified as incidentally detected on routine imaging scans for cancer staging or evaluation of cancer treatment response [8].

International guidelines suggest the same initial and long-term treatment for cancer patients with incidental VTE as for those with symptomatic VTE, mainly based on the comparable risk of recurrent VTE observed in retrospective cohort studies [9-11]. A recent prospective cohort study of 695 cancer patients with incidental pulmonary embolism reported a 1-year incidence of recurrent VTE of $6 \%$, confirming the clinical relevance of these events [12]. Translating these results to current clinical practice is challenging because some patients received subtherapeutic doses of anticoagulants, only $5 \%$ received a direct oral anticoagulant and a control group with symptomatic VTE was not included.

The Hokusai VTE Cancer Study was the first randomised controlled trial evaluating treatment of cancer-associated VTE, which also included patients with incidental VTE [13]. Therefore, it provides a unique opportunity to compare clinical outcomes in patients with incidental and symptomatic VTE, evaluate the safety and efficacy of edoxaban versus dalteparin in these groups, and investigate risk factors for recurrent VTE in patients with incidental VTE.

\section{Methods \\ Patients}

The Hokusai VTE Cancer Study was an open-label trial that randomised 1050 cancer patients with symptomatic or incidentally detected VTE to either oral edoxaban or subcutaneous dalteparin once daily. Patients with cancer-associated VTE were randomised in a 1:1 ratio with the use of an interactive Web-based system with stratification of bleeding risk factors and the requirement of anticoagulant dosage reduction. The treatment duration was 6-12 months, at the discretion of the treating physician. Both VTE and recurrent VTE were considered incidental if diagnosed on radiological imaging tests performed for reasons other than a clinical suspicion of VTE. The sample size of the trial was based on the assumption of equal effectives of dalteparin and edoxaban in cancer patients and a rate of the primary outcome of $20 \%$ at 12 months of follow-up. The full list of inclusion and exclusion criteria, study protocol, and detailed description of the study design were reported previously (ClinicalTrials.gov identifier NCT02073682) [13, 14]. The CONSORT checklist and flow diagram are shown in supplementary figure S1.

All patients provided written informed consent and the study was approved by the ethical committees of participating centres.

The present post hoc analysis of this trial was restricted to patients who received at least one dose of study drug and had their index event centrally adjudicated by an independent radiologist, blinded to treatment allocation. The radiologist also evaluated the thrombus location for each DVT and pulmonary embolism, and assessed the extent of pulmonary embolism based on a predefined perfusion score (supplementary material) [15].

\section{Outcomes}

The primary outcome of the current study was the composite of first recurrent VTE or major bleeding during the 12-month study period. Secondary outcomes included recurrent VTE, major bleeding and mortality during the 12-month study period. Additionally, outcomes were evaluated during the 6-month study period, which was the minimal treatment duration with the assigned study drug, and during the on-treatment period, defined as during treatment or up to 3 days after the last dose of study drug. Recurrent VTE was defined as a new symptomatic or incidental DVT or pulmonary embolism, fatal pulmonary embolism, or unexplained death for which pulmonary embolism could not be ruled out. Major

This article has an editorial commentary: https://doi.org/10.1183/13993003.00028-2020

This article has supplementary material available from erj.ersjournals.com

Data from the Hokusai VTE Cancer Study (ClinicalTrials.gov identifier NCT02073682) may be obtained by a third party upon reasonable request.

Received: 30 Aug 2019 | Accepted after revision: 20 Oct 2019 
bleeding was defined according to the criteria of the International Society on Thrombosis and Haemostasis [16] and the severity of major bleeding was classified in four categories according to a previously proposed method [17]. All study outcomes in the Hokusai VTE Cancer Study were adjudicated by an independent committee unaware of treatment allocation.

\section{Statistical analysis}

Differences between patients with incidental and symptomatic VTE were assessed using standard descriptive statistics. Cumulative incidences were presented as crude proportions and time-to-event curves were constructed using the Kaplan-Meier method. Hazard ratios were computed with $95 \%$ confidence intervals using a Cox proportional hazards model. In a second model, we adjusted for age, sex, index event (pulmonary embolism versus DVT), anticoagulant treatment (edoxaban versus dalteparin), Eastern Cooperative Oncology Group (ECOG) performance status (2 versus 0 or 1 ), cancer type (haematological, lung, breast, gastrointestinal, urogenital, gynaecological or other), previous VTE and tumour stage (distant metastasis versus other). For patients with incidental VTE, risk factors for recurrent VTE were assessed in a univariable Cox proportional hazards model. In a sensitivity analysis, time-to-event curves of the main study outcomes were constructed with the competing risk approach proposed by FINE and GRAY [18]. Likewise, the association between risk factors and recurrent VTE in patients with incidental VTE were additionally evaluated by computing subdistribution hazard ratios with the competing risk approach. A p-value $<0.05$ was considered statistically significant. Statistical analyses were performed in $\mathrm{R}$ version 3.5.1 (www.R-project.org).

\section{Results}

\section{Patients with incidental VTE}

Of the 1046 cancer patients enrolled in the Hokusai VTE Cancer Study, 340 (32\%) had a reported incidental VTE, which was radiologically confirmed in 331 patients. Baseline characteristics are shown in table 1. 16 patients (4.8\%) had haematological cancer and 315 patients (95.2\%) had a solid tumour. The index event was incidental pulmonary embolism with or without DVT in 290 patients (87.6\%) and incidental DVT alone in 41 patients (12.4\%). Of the 290 patients with an incidental pulmonary embolism, the anatomical extent was considered limited in 112 patients (38.6\%), intermediate in 132 patients (45.5\%) and extensive in 46 patients (15.9\%).

The primary outcome, which was the composite of first recurrent VTE or major bleeding during the 12-month study period, occurred in 42 patients (12.7\%) (figure 1a and table 2). 26 patients (7.9\%) had recurrent VTE (figure 1b), of whom 16 patients (4.8\%) had recurrent pulmonary embolism with or without DVT and 10 patients (3.0\%) had recurrent DVT only. Of the 16 patients with recurrent pulmonary embolism, seven (43.8\%) were symptomatic, five (31.3\%) were incidental and there were four (25.0\%) deaths for which pulmonary embolism could not be ruled out. Of the 10 recurrent DVT events, six (60\%) were symptomatic and four (40\%) were incidental. On-treatment recurrent VTE occurred in 19 patients (5.7\%). Major bleeding occurred in 22 patients (6.6\%) (figure 1c) and on-treatment major bleeding occurred in 20 patients $(6.0 \%)$, of which nine patients (45\%) had a severe clinical bleeding presentation (category 3 or 4 ). During the 12-month study period, 123 patients (37.2\%) with incidental VTE died (supplementary figure S2). The sensitivity analysis restricted to study outcomes during the first 6 months of follow-up was comparable to the primary analysis outcome and is presented in supplementary table S1. The competing risk approach showed similar results (supplementary figure S5a-c).

\section{Patients with symptomatic VTE}

Symptomatic VTE was the qualifying event in 706 patients; 679 events were radiologically confirmed centrally. Baseline characteristics of patients with symptomatic VTE are shown in table 1. The symptomatic index event was pulmonary embolism with or without DVT in 353 patients (52.0\%) and DVT only in 326 patients $(48.0 \%)$. Of the 353 patients with symptomatic pulmonary embolism, the anatomical extent was considered limited in 117 patients (33.1\%), intermediate in 129 patients (36.5\%) and extensive in 107 patients (30.3\%).

The primary outcome occurred in 94 patients (13.8\%) during the 12-month study period (figure 1a). Recurrent VTE occurred in 74 patients (10.9\%), of whom 39 patients (5.7\%) had recurrent pulmonary embolism with or without DVT and 35 patients (5.2\%) had recurrent DVT alone (figure 1b). Of the 39 recurrent pulmonary embolism events, 23 (58.9\%) were symptomatic, 10 (25.6\%) were incidental and six (15.4\%) were deaths for which pulmonary embolism could not be ruled out. Of the 35 recurrent DVT events, 32 (91.4\%) were symptomatic and three (8.6\%) were incidental. On-treatment recurrent VTE occurred in 53 patients (7.8\%). Major bleeding occurred in 33 patients (4.9\%) and on-treatment major bleeding occurred in 26 patients (3.8\%) (figure 1c), of which 12 patients (46.2\%) had a severe presentation 


\begin{tabular}{|c|c|c|c|}
\hline & Incidental VTE & Symptomatic VTE & p-value \\
\hline Patients & 331 & 679 & \\
\hline Age years & $63.0 \pm 11.6$ & $64.5 \pm 11.3$ & 0.060 \\
\hline Male & $161(48.6)$ & $361(53.2)$ & 0.199 \\
\hline \multicolumn{4}{|l|}{ Criteria for edoxaban dose reduction } \\
\hline Body weight $\leqslant 60 \mathrm{~kg}$ & $54(16.3)$ & $102(15.0)$ & 0.659 \\
\hline Creatinine clearance $30-50 \mathrm{~mL} \cdot \mathrm{min}^{-1}$ & $20(6.0)$ & $50(7.4)$ & 0.519 \\
\hline Receiving potent $\mathrm{P}$-glycoprotein inhibitors & $5(1.5)$ & $31(4.6)$ & 0.023 \\
\hline Previous VTE & $30(9.1)$ & $82(12.1)$ & 0.185 \\
\hline Qualifying VTE event & & & $<0.001$ \\
\hline Pulmonary embolism with or without DVT & $290(87.6)$ & $353(52.0)$ & \\
\hline DVT only & $41(12.4)$ & $326(48.0)$ & \\
\hline Most proximal extent of pulmonary embolism & & & $<0.001$ \\
\hline Limited & $112(38.6)$ & $117(33.1)$ & \\
\hline Intermediate & $132(45.5)$ & $129(36.5)$ & \\
\hline Extensive & $46(15.9)$ & $107(30.3)$ & \\
\hline ECOG performance status & & & 0.066 \\
\hline 0 & $111(33.5)$ & $180(26.5)$ & \\
\hline 1 & $152(45.9)$ & $321(47.3)$ & \\
\hline 2 & $66(19.9)$ & $175(25.8)$ & \\
\hline Unknown & $2(0.6)$ & $3(0.4)$ & \\
\hline Haematological cancer & $16(4.8)$ & $91(13.4)$ & $<0.001$ \\
\hline Solid cancer & 315 (95.2) & $588(86.6)$ & $<0.001$ \\
\hline Urogenital & $40(12.1)$ & $95(14.0)$ & \\
\hline Colorectal & $63(19.0)$ & $92(13.5)$ & \\
\hline Hepatobiliary and pancreatic & $34(10.3)$ & $52(7.7)$ & \\
\hline Upper gastrointestinal & $20(6.0)$ & $33(4.9)$ & \\
\hline Lung & $50(15.1)$ & $94(13.8)$ & \\
\hline Gynaecological & $43(13.0)$ & $66(9.7)$ & \\
\hline Breast & $33(10.0)$ & $87(12.8)$ & \\
\hline Other & $32(9.7)$ & $69(10.2)$ & \\
\hline Solid and haematological & 1 & 2 & \\
\hline Solid cancer stage & & & $<0.001$ \\
\hline Local disease & $15(4.8)$ & $26(4.4)$ & \\
\hline Locoregional disease & $20(6.4)$ & $38(6.5)$ & \\
\hline Locoregional recurrence & $11(3.5)$ & $27(4.6)$ & \\
\hline Metastatic disease & $209(66.4)$ & $324(55.1)$ & \\
\hline Not available & $60(19.1)$ & $173(29.4)$ & \\
\hline Cancer treatment within previous 4 weeks ${ }^{\#}$ & 236 (71.3) & $492(72.5)$ & 0.756 \\
\hline Anticoagulant treatment dalteparin & $171(51.7)$ & $337(49.6)$ & 0.590 \\
\hline Anticoagulant treatment duration days & 195 (92-352) & $189(76-353)$ & 0.498 \\
\hline
\end{tabular}

Data are presented as $\mathrm{n}$, mean \pm SD, $\mathrm{n}(\%)$ or median (interquartile range), unless otherwise stated. DVT: deep-vein thrombosis; ECOG: Eastern Cooperative Oncology Group. ${ }^{\#}$ : patients could receive more than one anticancer drug; numbers do not include cancer therapy initiated after randomisation.

(category 3 or 4). Study outcomes during the 12-month study period are presented in table 2 . Supplementary table S1 shows outcomes during the 6-month study period. The competing risk analysis showed comparable results (supplementary figure S5a-c).

\section{Outcomes in patients with incidental versus symptomatic VTE}

Dalteparin was given to 171 patients (51.7\%) with incidental VTE and 337 patients (49.6\%) with symptomatic VTE (table 1). The median (interquartile range) anticoagulant duration was 195 (92352) days in those with incidental VTE and 189 (76-353) days in those with symptomatic VTE ( $\mathrm{p}=0.50)$. More patients had pulmonary embolism with or without DVT as the qualifying index event in the group with incidental VTE $(87.6 \%)$ compared with the group with symptomatic VTE $(52.0 \%)(\mathrm{p}<0.001)$. While the thrombus was considered extensive in $15.9 \%$ of patients with incidental pulmonary embolism, it was considered extensive in $30.3 \%$ of patients with symptomatic pulmonary embolism $(\mathrm{p}<0.001)$. Fewer patients with incidental VTE had haematological cancer compared with those with symptomatic VTE $(4.8 \%$ versus $13.4 \%)(\mathrm{p}<0.001)$. 


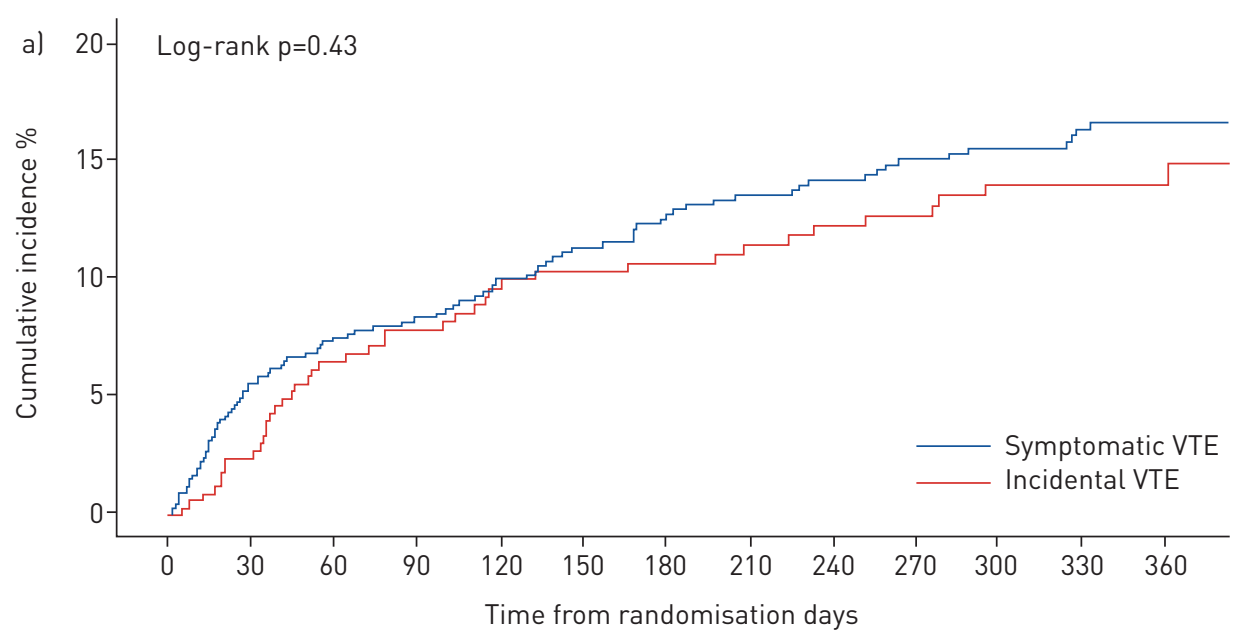

At risk $n$ :

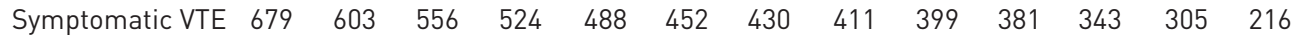

$\begin{array}{llllllllllllll}\text { Incidental VTE } & 331 & 313 & 289 & 271 & 255 & 245 & 240 & 229 & 212 & 205 & 183 & 157 & 103\end{array}$

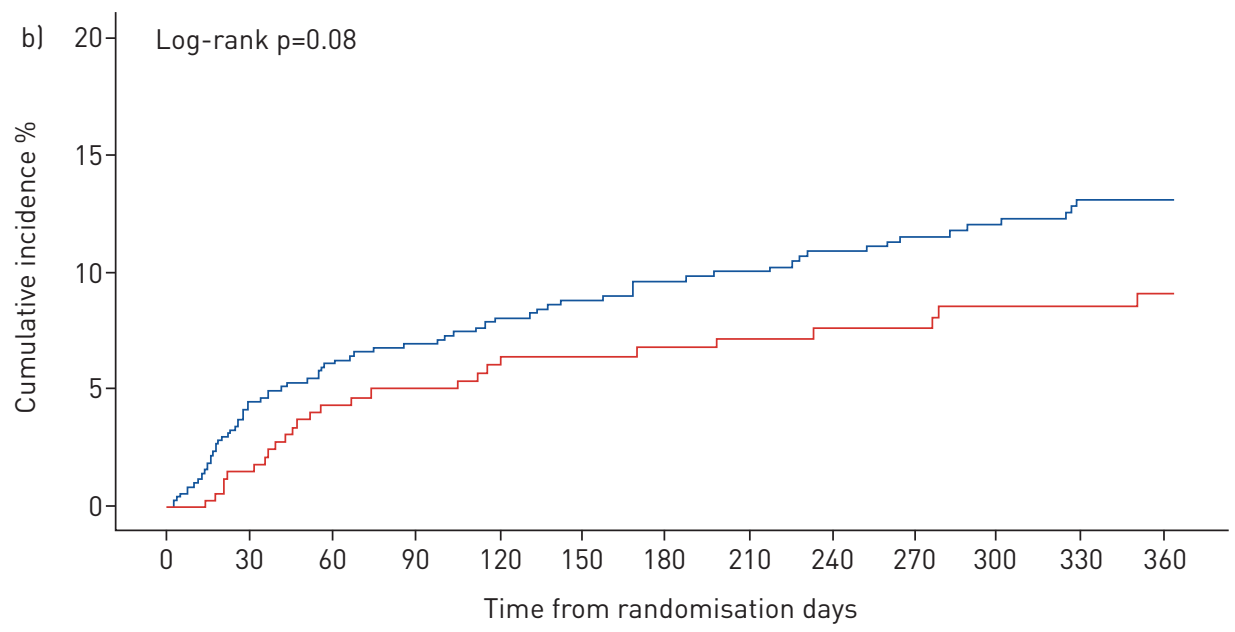

At risk $n$ :

$\begin{array}{llllllllllllll}\text { Symptomatic VTE } & 679 & 615 & 561 & 530 & 494 & 461 & 442 & 426 & 410 & 392 & 352 & 310 & 219 \\ \text { Incidental VTE } & 331 & 317 & 294 & 277 & 261 & 252 & 246 & 237 & 220 & 214 & 191 & 165 & 110\end{array}$

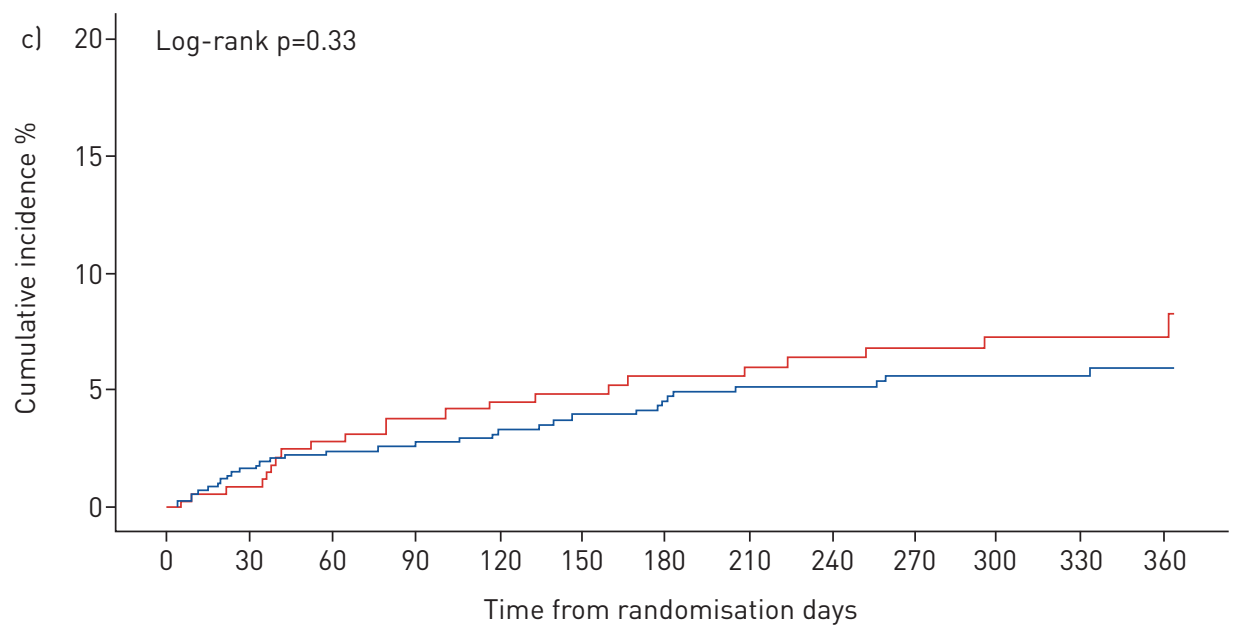

At risk n:

$\begin{array}{llllllllllllll}\text { Symptomatic VTE } & 679 & 628 & 583 & 551 & 518 & 482 & 462 & 443 & 427 & 409 & 370 & 331 & 235\end{array}$

$\begin{array}{llllllllllllll}\text { Incidental VTE } & 331 & 318 & 297 & 280 & 267 & 256 & 249 & 240 & 219 & 212 & 191 & 163 & 108\end{array}$

FIGURE 1 Cumulative incidence of a) primary outcome (first recurrent venous thromboembolism (VTE) or major bleeding), b) recurrent VTE and c) major bleeding in incidental VTE versus symptomatic VTE. 
TABLE 2 Outcomes in patients with incidental and symptomatic venous thromboembolism (VTE) during the 12-month study period

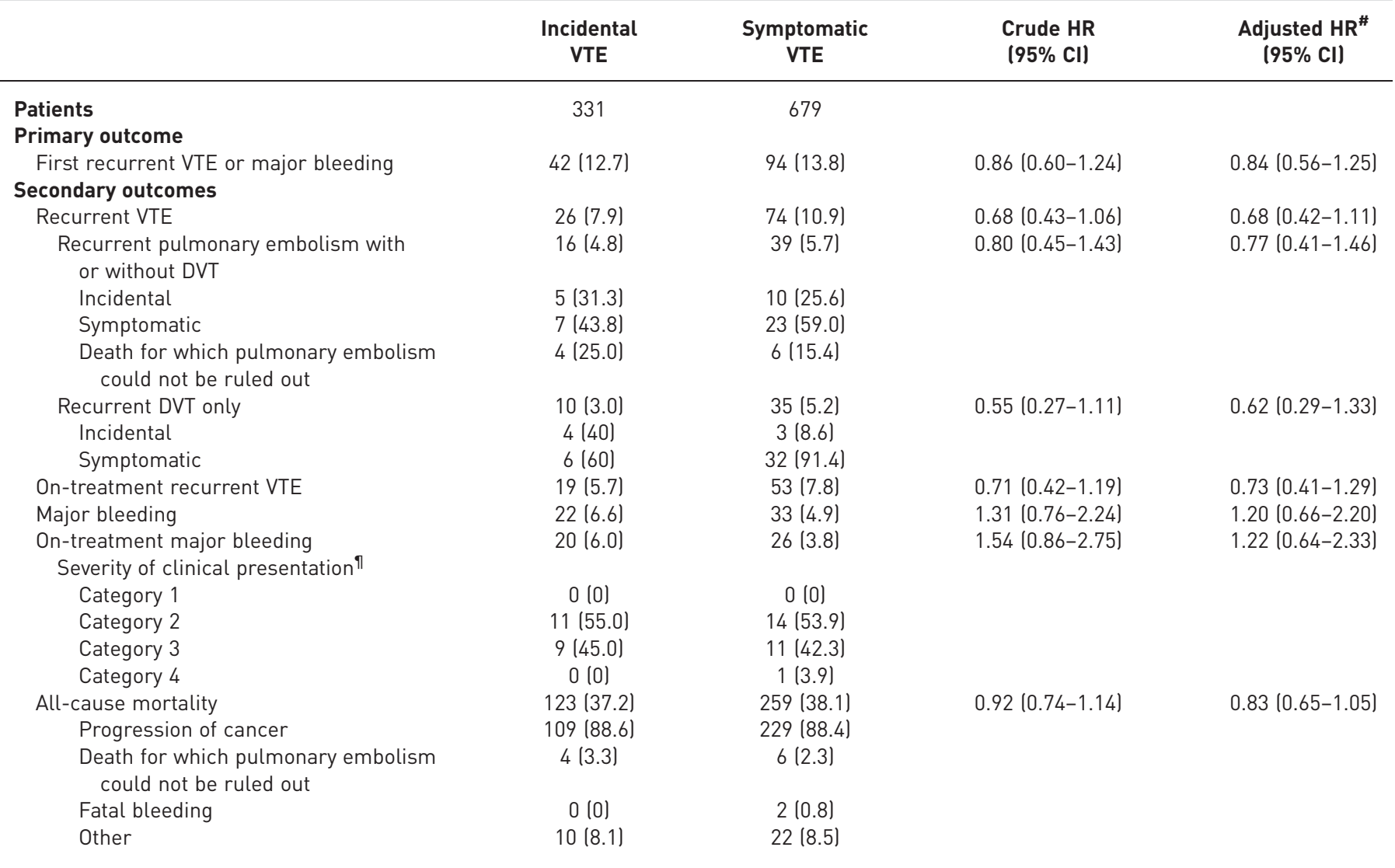

Data are presented as $\mathrm{n}$ or $\mathrm{n}(\%)$, unless otherwise stated. HR: hazard ratio; DVT: deep-vein thrombosis. ${ }^{\#}$ : adjusted for age, sex, anticoagulant treatment, Eastern Cooperative Oncology Group performance status (2 versus lower), cancer type (haematological, breast, lung, urogenital, gastrointestinal, gynaecological or other) and tumour stage (distant metastasis versus local/locoregional disease or haematological). The analysis of recurrent VTE events was additionally adjusted for prior VTE and the analysis of major bleeding events was additionally adjusted for the presence of any bleeding risk factor. Risk factors for bleeding included the use of antiplatelet therapy, the presence of brain tumour, bevacizumab in the 6 weeks prior to randomisation, regionally advanced or metastatic disease, gastrointestinal or urothelial cancer. १: classification of the clinical presentation of major bleeding events. Category 1: bleeding events presenting without any clinical emergency; Category 2: all bleeding events that could not be classified to any of the other three categories, as they presented with the need for some measures but without clear urgency; Category 3: bleeding events presenting with great medical emergency; for example, with haemodynamic instability; or cerebral bleeding presenting with neurological symptoms; Category 4: bleeding events that are fatal before or almost immediately upon entering the hospital.

During the complete study period, there was no statistically significant difference between patients with incidental and symptomatic VTE in the risk of the primary outcome (crude HR 0.86, 95\% CI 0.60-1.24; adjusted HR $0.84,95 \%$ CI 0.56-1.25) (figure 1), recurrent VTE (crude HR 0.68, 95\% CI 0.43-1.06; adjusted HR $0.68,95 \%$ CI $0.42-1.11$ ) and on-treatment major bleeding (crude HR 1.54, 95\% CI 0.86-2.75; adjusted HR 1.22, 95\% CI 0.64-2.33) (table 2). All-cause mortality after 12 months was comparable in patients with incidental and symptomatic VTE (37.2\% and 38.1\%, respectively; crude HR 0.92, 95\% CI 0.74-1.14; adjusted HR 0.83, 95\% CI 0.65-1.05).

\section{Edoxaban versus dalteparin}

In patients with incidental VTE, the primary outcome occurred in 19 out of 160 patients (11.9\%) randomised to edoxaban and in 23 out of 171 patients (13.5\%) who received dalteparin (HR 0.94, 95\% CI 0.51-1.73) (supplementary figure S3). In patients with symptomatic VTE, the primary outcome occurred in 46 out of 355 patients $(13.5 \%)$ in the edoxaban group and 48 out of 351 patients (14.2\%) in the dalteparin group (HR 0.95, 95\% CI 0.63-1.42) (supplementary figure S4). There was no evidence of effect modification of incidental versus symptomatic VTE in the occurrence of the primary outcome ( $p_{\text {interaction }}=0.97$ ). There were no significant differences in any of the other study outcomes after 12 months of follow-up (supplementary table S2). 
Risk factors for recurrent VTE in patients with incidental VTE

In patients with incidental VTE, age, male sex, anatomical extent of pulmonary embolism or body mass index were not associated with the risk of recurrent VTE (table 3). Patients with a decreased performance status were more likely to experience recurrent VTE during 12 months of follow-up (ECOG performance status 2 versus 0: HR 5.24, 95\% CI 1.81-15.18). The subdistribution hazard ratios estimated with the competing risk approach yielded similar results (table 3 ).

\section{Discussion}

The present study evaluated clinical outcomes in cancer patients with incidental and symptomatic VTE using data from the Hokusai VTE Cancer Study. The main finding is that the risk of recurrent VTE in patients with incidental VTE was significant: $7.9 \%$ of patients with incidental VTE developed recurrent VTE during 12 months of follow-up despite initiating anticoagulant treatment. Approximately half of the recurrent VTE events in patients with incidental VTE were symptomatic and a quarter were deaths for which pulmonary embolism could not be ruled out. These findings indicate that incidental VTE in cancer patients is a serious condition and support the current guidelines, which recommend that incidental VTE should be treated in the same manner as symptomatic VTE.

The detailed findings from the current analysis merit closer consideration. The 12-month risk of recurrent VTE was lower in patients with incidental VTE than in those with symptomatic VTE (7.9\% versus $10.9 \%$ ), although this difference was not statistically significant (HR 0.68, 95\% CI 0.43-1.06). A possible explanation could be differences in patient characteristics, which may impact the risk of VTE recurrence. For example, patients with symptomatic VTE more frequently had extensive pulmonary embolism $(30.3 \%$ versus $15.9 \%$; $\mathrm{p}<0.001)$ and poor ECOG performance status $(25.8 \%$ versus $19.9 \%$; $\mathrm{p}=0.066)$ (table 1$)$, which are potential risk factors for recurrence. Also, fewer patients with incidental VTE had haematological cancer, presumably because routine imaging for monitoring of cancer progression, on which incidental findings are detected, is less frequently used in these patients compared with those with solid cancers.

TABLE 3 Risk factors for recurrent venous thromboembolism in patients with incidental venous thromboembolism (VTE)

\section{Variables at study randomisation}

Univariable HR

(95\% Cl)

$0.95(0.81-1.12)$

$1.18(0.54-2.55)$

$1.16(0.86-1.56)$

Male sex

Body mass index (per $5 \mathrm{~kg} \cdot \mathrm{m}^{-2}$ increase)

Most proximal extent of pulmonary embolism

Limited

Intermediate

Extensive

Perfusion score (log)

Creatinine clearance $<60 \mathrm{~mL} \cdot \mathrm{min}^{-1}$

Leukocyte count $>11 \mathrm{~mm}^{-3}$

Platelet count $\geqslant 350 \mathrm{~mm}^{-3}$

ECOG performance status

0

1

2

\section{Primary tumour site}

Breast

Lung

Gastrointestinal

Gynaecological

Haematological

Urogenital

Metastatic versus nonmetastatic disease

Received radiotherapy 4 weeks before randomisation

Received chemotherapy 4 weeks before randomisation

Received hormonal therapy 4 weeks before randomisation

Previous VTE

Anticoagulant treatment: dalteparin versus edoxaban
Reference

$0.83(0.36-1.91)$

$0.43(0.10-1.93)$

$3.07(0.15-64.16)$

$0.31(0.07-1.31)$

$1.67(0.58-4.85)$

$2.01(0.76-5.33)$

Reference

$1.57(0.54-4.60)$

$5.24(1.81-15.18)$

Reference
$1.51(0.28-8.26)$
$1.08(0.22-5.19)$
$1.99(0.39-10.24)$
NA
$2.61(0.53-12.96)$
$2.56(0.96-6.79)$
$3.82(0.90-16.14)$
$1.17(0.52-2.62)$
$1.05(0.25-4.38)$
$1.26(0.378-4.19)$
$1.99(0.87-4.58)$

Subdistribution HR $(95 \% \mathrm{Cl})$

$0.96(0.81-1.14)$

$1.23(0.57-2.66)$

$1.19(0.83-1.71)$

Reference $0.84(0.367-1.94)$

$0.42(0.09-1.87)$

$3.42(0.24-48.12)$

$0.29(0.07-1.25)$

$1.44(0.49-4.21)$

$1.87(0.71-4.92)$

Reference

1.50 (0.52-4.37)

$4.02(1.41-11.49)$

Reference

$1.35(0.26-7.16)$

$1.01(0.21-4.82)$

$2.00(0.40-9.90)$

NA

$2.65(0.55-12.75)$

$2.25(0.86-5.90)$

$3.62(0.88-14.84)$

$1.16(0.52-2.59)$

$1.10(0.27-4.56)$

$1.33(0.40-4.37)$

$2.13(0.92-4.92)$

ECOG: Eastern Cooperative Oncology Group; NA: not applicable. 
Nonetheless, there were no substantial changes in the hazard ratios for recurrent VTE after adjusting for these confounding factors, suggesting that symptomatic VTE may be associated with an inherently higher risk of recurrence. Conversely, the 12-month major bleeding risk was numerically higher in patients with incidental VTE (6.6\%) than in those with symptomatic VTE (4.9\%) (HR 1.31, 95\% CI 0.76-2.24). This difference could be explained by the higher prevalence of gastrointestinal cancer in patients with incidental VTE (35.4\% versus $26.1 \%$ ), a cancer type found to be associated with an increased risk of major bleeding $[19,20]$. There was no difference in overall survival between patients with incidental and symptomatic VTE, which is in line with the findings of several other studies [8, 21-23]. In the Hokusai VTE Cancer Study, the decision to continue or discontinue anticoagulation after 6 months of follow-up was at the discretion of the treating clinician [13]. The median duration of anticoagulant treatment during total follow-up was similar between patients with incidental VTE and symptomatic VTE (195 versus 189 days). This indicates that most of the clinicians in this study adhered to the guidelines, which suggests similar treatment durations for patients with incidental or symptomatic VTE. Within the groups of incidental and symptomatic VTE, there was no difference in the occurrence of the primary outcome between patients randomised to edoxaban and dalteparin, which confirms that oral edoxaban is an acceptable alternative to injectable dalteparin in both patient groups.

Several studies previously evaluated clinical outcomes in cancer patients with incidental VTE. In line with our findings, a prospective cohort study of 695 cancer patients with incidental pulmonary embolism reported 12-month incidences of recurrent VTE and on-treatment major bleeding of $6.0 \%$ and $5.8 \%$, respectively [12]. Comparable results were obtained in an individual patient data meta-analysis of mostly retrospective data, comprising 11 cohort studies and 926 cancer patients, which reported a pooled 6-month risk of 5.8\% for recurrent VTE and 4.7\% for major bleeding [24]. However, Den ExTER et al. [22] observed a much higher 12-month recurrent VTE risk of $13.3 \%$ in a small retrospective cohort study of 51 patients with incidental pulmonary embolism. Higher rates of recurrent VTE in patients with incidental VTE were also observed in studies by Font et al. [8] and Aмато et al. [25]. Potential explanations for these differences include the larger proportion of patients receiving vitamin $\mathrm{K}$ antagonists in these older studies, a treatment known to be associated with a higher risk of recurrence, misclassification of incidental VTE or study outcomes and enrolment of cancer types with a higher risk of recurrence. In contrast, we present findings from a prospective, randomised controlled trial with strict adjudication of index event and study outcome events by specialists blinded to treatment allocation.

Few studies previously compared outcomes between patients with incidental and symptomatic VTE, reporting conflicting findings. The study by DEN ExTER et al. [22] demonstrated that the risk of recurrence is comparable in both groups. Recently, the pilot SELECT-D study compared rivaroxaban with dalteparin in 406 patients with cancer-associated VTE, of whom 213 patients (52.5\%) had incidental pulmonary embolism or DVT at baseline [20]. The authors reported that the risk of recurrent VTE was significantly higher in patients with symptomatic VTE than in those with incidental VTE (HR 2.8, 95\% CI 1.2-6.4). Differences between our results and those of the SELECT-D study may reflect the smaller sample size, the higher proportion of incidental VTE and the shorter reported follow-up duration of 6 months [20].

We have investigated potential risk factors for predicting recurrent VTE in patients with incidental VTE (table 3) and identified only a poor ECOG performance status of $\geqslant 2$. Both the perfusion score and anatomical pulmonary embolism clot extent were not significantly associated with recurrent VTE in patients with incidental pulmonary embolism (extensive versus limited clot: HR 0.43 , 95\% CI 0.10-1.93; perfusion score: HR 3.07, 95\% CI 0.15-62.2).

The strengths of the current analysis include the complete follow-up of almost all randomised patients [13]. In contrast to previous studies, anticoagulant treatment was uniform in patients with incidental and symptomatic VTE, and outcomes were adjudicated by an independent committee unaware of treatment allocation, thus minimising observer bias. Several limitations need to be taken into consideration. Although the open-label design could have influenced treatment duration in patients with symptomatic and incidental VTE, there were no differences between these groups. It should be acknowledged that incidental VTE is not necessarily asymptomatic. However, we followed the terminology and definition recommended by the International Society on Thrombosis and Haemostasis [26]. Since the Hokusai VTE Cancer Study was not primarily designed for the current research question, power to detect significant differences may have been limited. Although hazard ratios were adjusted for several potential confounders, we cannot exclude residual confounding. Since death is a competing risk for the occurrence of study events, Kaplan-Meier curves could have led to overestimating the cumulative incidence of study outcomes. However, a competing risk approach yielded similar results.

The improved quality and routine utilisation of radiological imaging in cancer patients frequently lead to the diagnosis of incidental VTE. Our findings substantiate the high risk of recurrent VTE despite 
anticoagulant treatment in patients with incidental events and the results strengthen current guidelines, which recommend the same treatment for incidental VTE as is used for symptomatic VTE. Future randomised trials evaluating anticoagulant treatment in cancer patients should include patients with incidental VTE by default. Additional risk factors for incidental VTE need to be identified in order to better individualise anticoagulant treatment for this important patient group.

Author contributions: Study conception and design: F.I. Mulder, N. van Es, M. Di Nisio, C. Ay, H.R. Büller. Data acquisition: M. Di Nisio, M. Carrier, A. Segers, P. Verhamme, T. Wang, J.I. Weitz, S. Middeldorp, G. Raskob, L.F.M. Beenen, H.R. Büller, N. van Es. Statistical analysis: F.I. Mulder, N. van Es, G. Zhang. Interpretation of the data: all authors. Drafting of the manuscript: all authors. Critical revision of the manuscript for important intellectual content: all authors. Final approval of the manuscript: all authors. H.R. Büller is guarantor and takes responsibility for the integrity of the work as a whole.

Conflict of interest: F.I. Mulder has nothing to disclose. M. Di Nisio reports personal fees from Daiichi Sankyo outside the submitted work. C. Ay reports personal fees from Bayer, Daiichi Sankyo, Pfizer and BMS, outside the submitted work. M. Carrier reports personal fees from Daiichi Sankyo during the conduct of the study; grants and personal fees from BMS, LEO Pharma; personal fees from Pfizer, Sanofi and Bayer outside the submitted work. F.T.M. Bosch has nothing to disclose. A. Segers reports grants from Daiichi Sankyo during the conduct of the study; grants from Ionis Pharmaceuticals, Daiichi Sankyo and Janssen Pharmaceuticals outside the submitted work. N. Kraaijpoel has nothing to disclose. M.A. Grosso reports personal fees from Daiichi Sankyo outside the submitted work. G. Zhang reports personal fees from Daiichi Sankyo outside the submitted work. P. Verhamme reports grants and personal fees from Daiichi Sankyo, during the conduct of the study; grants and personal fees from Bayer Healthcare, Boehringer Ingelheim and LEO Pharma; personal fees from BMS, Portola, Medscape, Medtronic and Pfizer; and grants from Sanofi outside the submitted work. T-Z. Wang reports nonfinancial support from Daiichi Sankyo during the conduct of the study. J.I. Weitz reports personal fees from Daiichi Sankyo, during the conduct of the study; personal fees from Bayer Healthcare, BMS, Boehringer Ingelheim, Ionis Pharmaceuticals, Janssen, Johnson \& Johnson, Pfizer, Portola, Medscape and Novartis outside the submitted work. S. Middeldorp reports grants and personal fees from GSK, BMS/Pfizer, Aspen, Daiichi Sankyo; personal fees from Bayer, Boehringer Ingelheim; and grants from Sanquin. G. Raskob reports personal fees from Daiichi Sankyo during the conduct of the study; personal fees from Bayer Healthcare, BMS, Boehringer Ingelheim, Eli Lilly, Janssen, Johnson \& Johnson, Pfizer, Portola, Merck and Medscape outside the submitted work. L.F.M. Beenen has nothing to disclose. H.R. Büller reports personal fees from Daiichi Sankyo, during the conduct of the study; personal fees from Bayer Healthcare, BMS/Pfizer, Boehringer Ingelheim, Portola, Medscape, Eli Lilly, Sanofi Aventis and Ionis outside the submitted work. N. van Es reports personal fees from Daiichi Sankyo during the conduct of the study; and personal fees from Pfizer outside the submitted work.

Support statement: This study received financial support from Daiichi Sankyo. Funding information for this article has been deposited with the Crossref Funder Registry.

\section{References}

1 Timp JF, Braekkan SK, Versteeg HH, et al. Epidemiology of cancer-associated venous thrombosis. Blood 2013; 122: $1712-1723$.

2 Khorana AA, Francis CW, Culakova E, et al. Thromboembolism is a leading cause of death in cancer patients receiving outpatient chemotherapy. J Thromb Haemost 2007; 5: 632-634.

3 Lloyd AJ, Dewilde S, Noble S, et al. What impact does venous thromboembolism and bleeding have on cancer patients' quality of life? Value Health 2018; 21: 449-455.

4 Khorana AA. Cancer-associated thrombosis: updates and controversies. Hematology Am Soc Hematol Educ Program 2012; 2012: 626-630.

5 Di Nisio M, Carrier M. Incidental venous thromboembolism: is anticoagulation indicated? Hematology Am Soc Hematol Educ Program 2017; 2017: 121-127.

6 Carrier M, Righini M, Wells PS, et al. Subsegmental pulmonary embolism diagnosed by computed tomography: incidence and clinical implications. A systematic review and meta-analysis of the management outcome studies. J Thromb Haemost 2010; 8: 1716-1722.

7 Wiener RS, Schwartz LM, Woloshin S. Time trends in pulmonary embolism in the United States: evidence of overdiagnosis. Arch Intern Med 2011; 171: 831-837.

8 Font C, Farrús B, Vidal L, et al. Incidental versus symptomatic venous thrombosis in cancer: a prospective observational study of 340 consecutive patients. Ann Oncol 2011; 22: 2101-2106.

9 Streiff MB, Holmstrom B, Ashrani A, et al. Cancer-associated venous thromboembolic disease, version 1.2015: featured updates to the NCCN Guidelines. J Natl Compr Cancer Netw 2015; 13: 1079-1095.

10 Lyman GH, Bohlke K, Khorana AA, et al. Venous thromboembolism prophylaxis and treatment in patients with cancer: American Society of Clinical Oncology clinical practice guideline update 2014. J Clin Oncol 2015; 33: 654-656.

11 Farge D, Bounameaux H, Brenner B, et al. International clinical practice guidelines including guidance for direct oral anticoagulants in the treatment and prophylaxis of venous thromboembolism in patients with cancer. Lancet Oncol 2016; 17: e452-e466.

12 Kraaijpoel N, Bleker SM, Meyer G, et al. Treatment and long-term clinical outcomes of incidental pulmonary embolism in patients with cancer: an international prospective cohort study. J Clin Oncol 2019; 37: 1713-1720.

13 Raskob GE, van Es N, Verhamme $\mathrm{P}$, et al. Edoxaban for the treatment of cancer-associated venous thromboembolism. N Engl J Med 2018; 378: 615-624.

14 van Es N, Di Nisio M, Bleker SM, et al. Edoxaban for treatment of venous thromboembolism in patients with cancer. Thromb Haemost 2015; 114: 1268-1276. 
15 Buller HR, Lensing AWA, Prins MH, et al. A dose-ranging study evaluating once-daily oral administration of the factor Xa inhibitor rivaroxaban in the treatment of patients with acute symptomatic deep vein thrombosis: the Einstein-DVT Dose-Ranging Study. Blood 2008; 112: 2242-2247.

16 Schulman S, Kearon C. Definition of major bleeding in clinical investigations of antihemostatic medicinal products in non-surgical patients. J Thromb Haemost 2005; 3: 692-694.

17 Bleker SM, Brekelmans MPA, Eerenberg ES, et al. Clinical impact of major bleeding in patients with venous thromboembolism treated with factor Xa inhibitors or vitamin $\mathrm{K}$ antagonists. An individual patient data meta-analysis. Thromb Haemost 2017; 117: 1944-1951.

18 Fine JP, Gray RJ. A proportional hazards model for the subdistribution of a competing risk. J Am Stat Assoc 1999; 94: 496-509.

19 Kraaijpoel N, Di Nisio M, Mulder FI, et al. Clinical impact of bleeding in cancer-associated venous thromboembolism: results from the Hokusai VTE Cancer Study. Thromb Haemost 2018; 118: 1439-1449.

20 Young AM, Marshall A, Thirlwall J, et al. Comparison of an oral factor Xa inhibitor with low molecular weight heparin in patients with cancer with venous thromboembolism: results of a randomized trial (SELECT-D). J Clin Oncol 2018; 36: 2017-2023.

21 Sahut D'Izarn M, Caumont Prim A, Planquette B, et al. Risk factors and clinical outcome of unsuspected pulmonary embolism in cancer patients: a case-control study. J Thromb Haemost 2012; 10: 2032-2038.

22 den Exter PL, Hooijer J, Dekkers OM, et al. Risk of recurrent venous thromboembolism and mortality in patients with cancer incidentally diagnosed with pulmonary embolism: a comparison with symptomatic patients. J Clin Oncol 2011; 29: 2405-2409.

23 Shinagare $\mathrm{AB}$, Okajima Y, Oxnard GR, et al. Unsuspected pulmonary embolism in lung cancer patients: comparison of clinical characteristics and outcome with suspected pulmonary embolism. Lung Cancer 2012; 78: $161-166$.

24 Van der Hulle T, den Exter PL, Planquette B, et al. Risk of recurrent venous thromboembolism and major hemorrhage in cancer-associated incidental pulmonary embolism among treated and untreated patients: a pooled analysis of 926 patients. J Thromb Haemost 2016; 14: 105-113.

25 Amato B, Compagna R, Rocca A, et al. Fondaparinux vs warfarin for the treatment of unsuspected pulmonary embolism in cancer patients. Drug Des Devel Ther 2016: 102041-102046.

26 Khorana AA, O'Connell C, Agnelli G, et al. Subcommittee on Hemostasis and Malignancy of the SSC of the ISTH. Incidental venous thromboembolism in oncology patients. J Thromb Haemost 2012; 10: 2602-2604. 